\title{
Contribuições para o desenho de estudos epidemiológicos sobre polvição por mercúrio na Amazônia*
}

\section{Contributions to the epidemiological study design on mercury pollution in Amazon}

\author{
Volney de Magalhães Câmara ${ }^{1,2}$ \\ ' Universidade Federal do Rio de Janeiro, Faculdade de Medicina, Rio de Janeiro, Rio de Janeiro, Brasil \\ 2 Universidade Federal do Rio de Janeiro, Instituto de Estudos em Saúde Coletiva, Rio de Janeiro, Rio de Janeiro, Brasil
}

Para alguns pesquisadores, o desenho de estudos epidemiológicos é considerado de elevada complexidade. Isso porque utiliza, em suas análises, estatísticas de diversos níveis, as quais avaliam a presença de determinados efeitos à saúde da população ou a baixa probabilidade de ocorrência, ao acaso, de associações entre a exposição a algum fator e o aparecimento de efeitos à saúde. Dada essa complexidade, um artigo de opinião sobre Epidemiologia, relacionada ao desenho de estudos sobre a exposição e efeitos do mercúrio na Amazônia, deve ter o principal objetivo de discutir pontos específicos dessa relação e/ou servir como uma aproximação inicial do leitor com esse tema tão relevante para a Saúde Coletiva. Estudos sobre mercúrio metálico e metilmercúrio, na Amazônia Legal, evidenciam pessoas expostas em áreas de garimpo, populações ribeirinhas, grupos indígenas e até residentes em áreas urbanas por meio da polvição intradomiciliar 1,2,3,4,

Uma das decisões preliminares, para a determinação do desenho das pesquisas, é a escolha de qual forma de mercúrio será o objeto do estudo. $O$ mercúrio metálico, lançado na atmosfera pela queima do amálgama ouro-mercúrio, e o metilmercúrio, que é originado por meio da cadeia biológica em situações específicas dos sedimentos dos rios, são completamente diferentes para o desenho dos estudos epidemiológicos. Amostras de urina são as mais utilizadas na avaliação dos níveis de exposição para a forma metálica; enquanto amostras de cabelo são preferenciais para a forma metilada.

A segunda decisão se refere ao tipo de estudo epidemiológico mais adequado. No caso do mercúrio, estudos descritivos, como o próprio título indica, descrevem como os efeitos do mercúrio ocorrem na população - ocorrência por sexo, idade, ocupação, tempo, local, entre outros. O outro tipo de estudo epidemiológico é o analítico, aquele que testa hipóteses que podem relacionar a exposição ao mercúrio com algum efeito à saúde (busca da relação causa-efeito), como, por exemplo, avaliar se a exposição ao mercúrio pode estar relacionada às alterações hematológicas ou a algum efeito específico na audição, visão, etc.

$\mathrm{Na}$ maioria das vezes, o pesquisador necessitará ir a campo para obter os níveis de exposição ao mercúrio e também os possíveis efeitos a serem investigados. Todavia, existem sistemas de informação disponíveis que podem auxiliar na busca de efeitos à saúde, como o Sistema de Informação sobre Mortalidade (SIM), o Sistema de Informações sobre Nascidos Vivos (SINASC); o Sistema Nacional de Informações Tóxico-Farmacológicas (Sinitox); o Sistema de Informação de Agravos de Notificação (Sinan) e o Sistema de Informações Hospitalares do Sistema Único de Saúde (SIHSUS). Outros, como a Pesquisa Nacional por Amostra de Domićlio do Instituto Brasileiro de Geografia e Estatística, incluem perguntas específicas sobre a morbidade da população. Para estudos na Amazônia, até por imensa área geográfica e pouca capilaridade do SUS, pode-se supor que os dados sobre efeitos na saúde da população dessa região, presentes nesses sistemas, não exprimam a realidade. A escolha do dado secundário ou primário é uma decisão do pesquisador, que deve avaliar qual o dado é mais viável e adequado para a sua pesquisab ${ }^{6}$.

Os estudos descritivos possuem elevada importância para a área de saúde e, evidentemente, para os estudos epidemiológicos. $\bigcirc$ que os profissionais dessa área conhecem atualmente sobre o quadro clínico da intoxicação por mercúrio, para definição de casos, são originados de muitos desses estudos.

Em relação às variáveis sobre as pessoas, essas pesquisas na Amazônia podem mostrar como diversas variáveis ocorrem na intoxicação; entre elas, as principais são sexo, idade, tipo de alimentação, profissão, hábitos culturais, tipo de religião, padrão de consumo (notadamente, nesse caso, torna-se relevante o consumo de pescado), renda, escolaridade, entre outras.

\footnotetext{
* Artigo de opinião escrito por Palestrante do II Encontro Científico Internacional do Instituto Evandro Chagas, com realização no período de 25 a 27 de outubro de 2017, em Ananindeua, Pará, Brasil. Todos os artigos dessa modalidade foram analisados pela Comissão Científica do Evento e, posteriormente, pelos Editores da RPAS.
}

\section{Correspondência / Correspondence:}

Volney de Magalhães Câmara

Universidade Federal do Rio de Janeiro, Faculdade de Medicina

Av. Brigadeiro Trompowsky, s/n; Praça da Prefeitura Universitária; llha do Fundão. Bairro: llha do Fundão. CEP: $21949-900$ - Rio de Janeiro, Rio de Janeiro, Brasil - Tel.: +55 (21) 2598-9328 
O tempo é outra variável relevante para qualquer estudo descritivo. Sabe-se que, na época das chuvas na Amazônia, é menor a exposição ao mercúrio metálico pela impossibilidade da atividade garimpeira em alguns locais. Uma intoxicação aguda, que pode ocorrer pela exposição elevada ao mercúrio metálico, pode apresentar casos de intoxicação em um curto espaço de tempo. Todavia, tanto para a forma metálica quanto para a metílica, os efeitos adversos à saúde relacionados ao mercúrio são de aparecimento crônico, muitas vezes, por exposição a esse metal em um longo período de tempo.

O local é outra variável importante, visto que pode direcionar esforços e, assim, maximizar atividades de vigilância para mitigação e prevenção de novos casos.
Os estudos epidemiológicos existentes, em sua maioria, são descritivos e, entre eles, aqueles que avaliam a prevalência dos efeitos do mercúrio na população. Como se pode observar no quadro 1, o estudo de incidência é de longa duração e, por esse motivo, mais difícil de ser realizado na exposição ao mercúrio ${ }^{7}$. Inicialmente, seleciona-se um grupo de pessoas a ser estudado, e, depois de um período de tempo determinado, observam-se todos os casos novos dos efeitos do mercúrio definidos para o estudo. Como esses efeitos são insidiosos e crônicos, deve-se esperar um longo tempo para que seja obtido um número relevante de casos. Já os estudos de prevalência são de curta duração e podem ser elaborados em até um dia, sendo mais viáveis e preferidos pelos pesquisadores. Nesses últimos, são incluídos todos os casos prevalentes, sejam novos ou antigos.

\begin{tabular}{|c|c|c|}
\hline Tipos & Exemplos de desenho do estudo & Factibilidade \\
\hline \multicolumn{3}{|c|}{ Observacionais/Descritivos } \\
\hline Incidência & $\begin{array}{l}\text { Identificação e acompanhamento de uma população exposta a um } \\
\text { polvente ambiental durante um período em que são registrados casos } \\
\text { novos de intoxicação pelo polvente que se quer estudar. }\end{array}$ & $\begin{array}{l}\text { Estudo de longa duração mais indicado para } \\
\text { as doenças agudas. }\end{array}$ \\
\hline Prevalência & $\begin{array}{l}\text { Identificação de uma população exposta a um polvente ambiental } \\
\text { e cálculo imediato de todos os casos existentes de intoxicação pelo } \\
\text { polvente. }\end{array}$ & $\begin{array}{l}\text { Estudo de curta duração e mais indicado para } \\
\text { as doenças crônicas. }\end{array}$ \\
\hline \multicolumn{3}{|c|}{ Observacionais/Associação entre variáveis } \\
\hline Ecológico & $\begin{array}{l}\text { Correlação entre os registros de uma determinada patologia e os de } \\
\text { níveis de exposição a um polvente por um período de tempo. }\end{array}$ & $\begin{array}{l}\text { Pouco factível devido à ausência de registros } \\
\text { dos polventes e da doença. }\end{array}$ \\
\hline $\begin{array}{l}\text { Seccional ou } \\
\text { Transversal }\end{array}$ & $\begin{array}{l}\text { Formar um grupo de pessoas expostas (censo ou uma amostra) ao } \\
\text { fator ambiental (Grupo Estudo) e outro grupo de pessoas não expostas } \\
\text { (Grupo Comparação). Logo após, comparar a prevalência dos efeitos } \\
\text { entre os dois grupos. }\end{array}$ & $\begin{array}{l}\text { Muito factível. Por ser de curta duração, é } \\
\text { indicado para efeitos crônicos. }\end{array}$ \\
\hline \multicolumn{3}{|c|}{ Observacionais/Comparativos/Evidência de causalidade } \\
\hline Cohort & $\begin{array}{l}\text { Inicialmente, formar dois grupos: Estudo e Comparação (igual aos } \\
\text { estudos seccionais); em seguida, comparar a incidência ou mortalidade } \\
\text { dos efeitos entre os dois grupos num determinado período. }\end{array}$ & $\begin{array}{l}\text { Pouco factível. Longa duração, indicado para } \\
\text { efeitos agudos e populações estáveis. }\end{array}$ \\
\hline Cohort histórico & $\begin{array}{l}\text { Por meio de dados de registro, os grupos de expostos (Estudo) e não } \\
\text { expostos (Comparação) são formados em um determinado momento } \\
\text { do passado. Logo após, compara-se as incidências/mortalidades do } \\
\text { efeito a ser estudado entre os dois grupos, desde o passado até o } \\
\text { momento atual. }\end{array}$ & Pouco factível pela ausência de registros. \\
\hline Caso-controle & $\begin{array}{l}\text { Forma-se um grupo de pessoas que apresentam os efeitos que se quer } \\
\text { estudar (Grupo Estudo) e outro grupo semelhante que não apresenta } \\
\text { esse efeito (Grupo Controle). O segundo passo é comparar, a proporção } \\
\text { de pessoas expostas no passado entre os dois grupos. }\end{array}$ & $\begin{array}{l}\text { Factível por ser de curta duração. Ideal para } \\
\text { doenças crônicas e de baixa prevalência. } \\
\text { Necessita de registros confiáveis do efeito. }\end{array}$ \\
\hline \multicolumn{3}{|c|}{ Intervencionais/Experimentais } \\
\hline Experimental & $\begin{array}{l}\text { Selecionar um grupo de pessoas que sofrerão um determinado tipo } \\
\text { de intervenção - por exemplo, o uso de novas drogas ou novas } \\
\text { tecnologias (Grupo Estudo) - e um grupo semelhante sem a intervenção } \\
\text { (Grupo Comparação); e acompanhar, por um período de tempo, para } \\
\text { comparar a incidência dos efeitos entre os dois grupos. }\end{array}$ & $\begin{array}{l}\text { Pouco factível devido a questões éticas. Ideal } \\
\text { para avaliar o impacto de um medicamento } \\
\text { a ser testado ou de um equipamento de } \\
\text { proteção no ambiente de trabalho. }\end{array}$ \\
\hline
\end{tabular}

Quadro 1 - Características selecionadas para o desenho dos estudos epidemiológicos em saúde ambiental e saúde do trabalhador 
O estudo do tipo ecológico é pouco viável para ser realizado no Brasil e particularmente na Amazônia. São necessários registros de exposição ao mercúrio e de casos de efeitos à saúde durante longos períodos de tempo, para que se calcule uma correlação entre essas duas variáveis (ver explicação no quadro 1). Entre as principais características dos estudos ecológicos, destaca-se o fato de não se incluir a pessoa individualmente para ser entrevistada ou avaliada clinicamente, mas sim populações, por meio das várias informações que se pode ter acesso nos sistemas citados anteriormente (mortalidade, morbidade e outras).

Os estudos analíticos são geralmente comparativos entre grupos segundo a exposição ou a presença de um efeito à saúde. $\bigcirc$ primeiro passo na realização de tais estudos, para qualquer pesquisador, é a definição da hipótese a ser testada. A hipótese alternativa é aquela que se deseja comprovar. Caso os testes estatísticos evidenciem alguma "diferença estatística", abandona-se a hipótese nula (quando não há diferença entre os grupos de estudo) e adota-se a hipótese alternativa. Os dois grupos necessitam ser o mais semelhantes possível e serem submetidos aos mesmos procedimentos. Isso é válido para todos os estudos analíticos. Deve-se evitar erros de seleção (ex.: escolha da população a ser estudada) e de medição (ex.: equipamentos não calibrados). A presença de variáveis de interferência (confounding) também distorce a associação exposição-doença. Como exemplos de vieses, pode-se citar: a) se no Grupo Estudo for incluída uma população mais idosa, uma maior proporção de efeitos neurológicos pode estar associada a esse perfil etário; b) o excesso de tremores de mãos em um grupo, ao invés de ser um indicativo de maior proporção de lesão neurológica, pode ter como origem o fato de um dos grupos incluir mais indivíduos alcoólatras.

estudo seccional é considerado por muitos autores como um estudo analítico. É talvez o mais factível para ser realizado. Inicialmente, são escolhidos os indivíduos do Grupo Estudo (nesse caso, os expostos ao mercúrio) e um Grupo Comparação, formado por não expostos (vale repetir que esses grupos devem ser o mais semelhantes possível). Logo após (podem ser poucos dias), é comparada a proporção de pessoas com efeitos entre os dois grupos, por meio de testes estatísticos, para avaliar a probabilidade de que essa diferença não seja por acaso. $\bigcirc$ problema dos estudos seccionais é que, no Grupo Estudo, podem estar incluídas pessoas que apresentaram efeitos a serem testados antes da exposição ao mercúrio e, assim, não poderiam ser associados a esse metal. Uma possibilidade, para minimização desse problema, poderia ser a utilização de um instrumento, como, por exemplo, um questionário bem elaborado, que pudesse identificar se o efeito surgiu após a exposição ao mercúrio.

estudo analítico cohort é o mais adequado para avaliar riscos, porque, ao acompanhar os grupos estudados, o pesquisador identificará os casos que surgiram após a exposição ao mercúrio. Todavia, é o mais difícil de ser realizado para estudos sobre esse metal em locais de grande movimento migratório, como a Amazônia. A seleção dos grupos Estudo e Comparação é semelhante ao estudo seccional; porém, a grande diferença é que são analisados, para comparação entre os grupos, apenas os casos novos que aparecem em um período de tempo definido. Uma avaliação dos estudos sobre polventes ambientais no Brasil apontou que poucos estudos, como o do tipo cohort, são realizados no país ${ }^{8}$.

Outra opção ainda mais difícil é cohort histórico ou cohort retrospectivo, em que, a partir de uma data no passado, são formados os grupos Estudo ou Comparação; e, a partir dessa data até o momento presente, são comparadas as proporções de efeitos.

Os estudos caso-controle são diferentes de todos os estudos analíticos que foram descritos anteriormente. É formado um Grupo Estudo de pessoas que apresentam o efeito relacionado ao mercúrio, que é o objeto de estudo, e um Grupo Controle com as que não apresentam esse tipo de efeito. Depois, as proporções de indivíduos que foram expostos ao mercúrio no passado são comparadas através de testes estatísticos entre esses dois grupos.

Finalmente, nos estudos experimentais, em que o pesquisador intervém, como, por exemplo, testando uma medicação para diminuir os efeitos do mercúrio, a questão da ética na pesquisa precisa ser avaliada com cautela. Deve-se esperar um longo tempo para sua aprovação, uma vez que é necessário ser encaminhado a instâncias superiores em Brasília.

Este é apenas um olhar superficial sobre os diversos tipos de estudos epidemiológicos que podem ser utilizados em pesquisas sobre o mercúrio na Amazônia. $\bigcirc$ pesquisador, de acordo com o efeito adverso à saúde a ser investigado, necessita escolher - tipo de estudo que possa responder à pesquisa, levando em consideração o tempo e os recursos materiais e humanos que estejam disponíveis. 


\section{REFERÊNCIAS}

1 Jesus IM, Brabo ES, Lima MO, Faial KRF, Moraes LLCS, Mendes RA, et al. Contribuição pioneira do Instituto Evandro Chagas para a saúde ambiental na Amazônia em 25 anos da Seção de Meio Ambiente. Rev Pan-Amaz Saude. 2016 dez;7 n. esp:83-92.

2 Passos CJS, Mergler D. Human mercury exposure and adverse health effects in the Amazon: a review. Cad Saude Publica. 2008;24 Suppl 4:S503-20.

3 Malm O, Dórea JG, Barbosa AC, Pinto FN, Weihe P. Sequential hair mercury in mothers and children from a traditional riverine population of the Rio Tapajós, Amazonia: seasonal changes. Environ Res. 2010 Oct;1 10(7):705-9.

4 Marques RC, Abreu L, Bernardi JVE, Dórea JG. Neurodevelopment of Amazonian children exposed to ethylmercury (from Thimerosal in vaccines) and methylmercury (from fish). Environ Res. 2016 Aug; 149:259-65.
5 Câmara VM, Tavares LMB, Filhote MIF, Malm O, Perez MA. A program for the control of indoor pollution by metallic mercury. Environ Res. 2000 Jun;83(2): 1 10-6.

6 Rothman KJ, Greenland S, Lash TL. Modern epidemiology. 3rd ed. Philadelphia: Lippincott Williams \& Wilkins; 2008.

7 Câmara VM, Tambellini AT. Considerações sobre o uso da epidemiologia nos estudos em saúde ambiental. Rev Bras Epidemiol. 2003 jun;6(2):95-104.

8 Froes Asmus CIR, Camara VM, Landrigan PJ, Claudio L. A systematic review of children's environmental health in Brazil. Ann Glob Health. 2016 Jan-Feb;82(1):132-48.

Recebido em / Received: 2/10/2017 Aceito em / Accepted: 11/10/2017 